\title{
Isoalliin-Derived Thiolanes Formed in Homogenized Onion.
}

Iveta Štefanová, ${ }^{\dagger}$ Jakub Zápal, ${ }^{\star}$ Martin Moos, ${ }^{\S}$ Marek Kuzma ${ }^{\ddagger}$, and Roman $\mathrm{Kubec}^{*}{ }^{\star \dagger}$

Department of Applied Chemistry, University of South Bohemia, Branišovská 31, 37005

České Budějovice, Czech Republic, Laboratory of Molecular Structure Characterization, Institute of Microbiology, The Czech Academy of Sciences, Vídeňská 1083, 14220 Prague 4, Czech Republic, Laboratory of Analytical Biochemistry, Biology Centre, The Czech Academy of Sciences, Branišovská 31, 370 05, České Budějovice, Czech Republic

*Tel.: +420-38-7772664. E-mail: kubecr@centrum.cz or kubec@ zf.jcu.cz.

${ }^{\dagger}$ University of South Bohemia.

$\$$ Laboratory of Molecular Structure Characterization.

$\S$ Biology Centre.

\section{CONTENT:}

- IUPAC names of cepadithiolactone A, onionins A-C, and cepathiolanes A-C.

- Representative chromatograms illustrating the isolation of cepadithiolactones A, onionins $\mathrm{A}-\mathrm{C}$, and cepathiolanes $\mathrm{A}-\mathrm{C}$ by preparative HPLC.

- $\mathrm{UV}$-vis spectrum of cepadithiolactone A.

- A comparison of the EI-MS spectrum of cepadithiolactone A with those reported for "zwiebelane isomer".

- MS/MS spectra of cepadithiolactones A, onionins A-C, and cepathiolanes A-C.

- IR spectra of cepadithiolactones A, onionins A, and cepathiolanes A.

- A picture of the pinking assay. 


\section{IUPAC names of the identified compounds}

cepadithiolactones A<smiles>CC1C(=S)SC(O)C1C</smiles>

onionins A

onionins $B$

onionins $\mathrm{C}$

cepathiolanes A<smiles>CC=CC([O-])SC1SC(O)C(C)C1C</smiles><smiles>CC1C(O)SC(S[SH](C)(=O)[O-])C1C</smiles>

cepathiolanes C<smiles>CC=C[O+]([O-])C1SC(O)C(C)C1C</smiles><smiles>CC1SC([S+](C)[O-])C(C)C1C</smiles><smiles>CCC[O+]([O-])C1SC(O)C(C)C1C</smiles>

cepathiolanes B

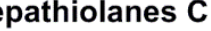

5-hydroxy-3,4-dimethyldihydrothiophene-2(3H)-thione

(E)-3,4-dimethyl-5-(prop-1-en-1-ylsulfinyl)tetrahydrothiophen-2-ol

3,4-dimethyl-5-(methylsulfinyl)tetrahydrothiophen-2-ol

3,4-dimethyl-5-(propylsulfinyl)tetrahydrothiophen-2-ol

(E)-S-(5-hydroxy-3,4-dimethyltetrahydrothiophen-2-yl) prop-1-ene-1-sulfinothioate

S-(5-hydroxy-3,4-dimethyltetrahydrothiophen-2-yl) methanesulfinothioate

S-(5-hydroxy-3,4-dimethyltetrahydrothiophen-2-yl) propane-1-sulfinothioate 


\section{Isolation of cepadithiolactones $A_{1-3}$ by preparative HPLC}
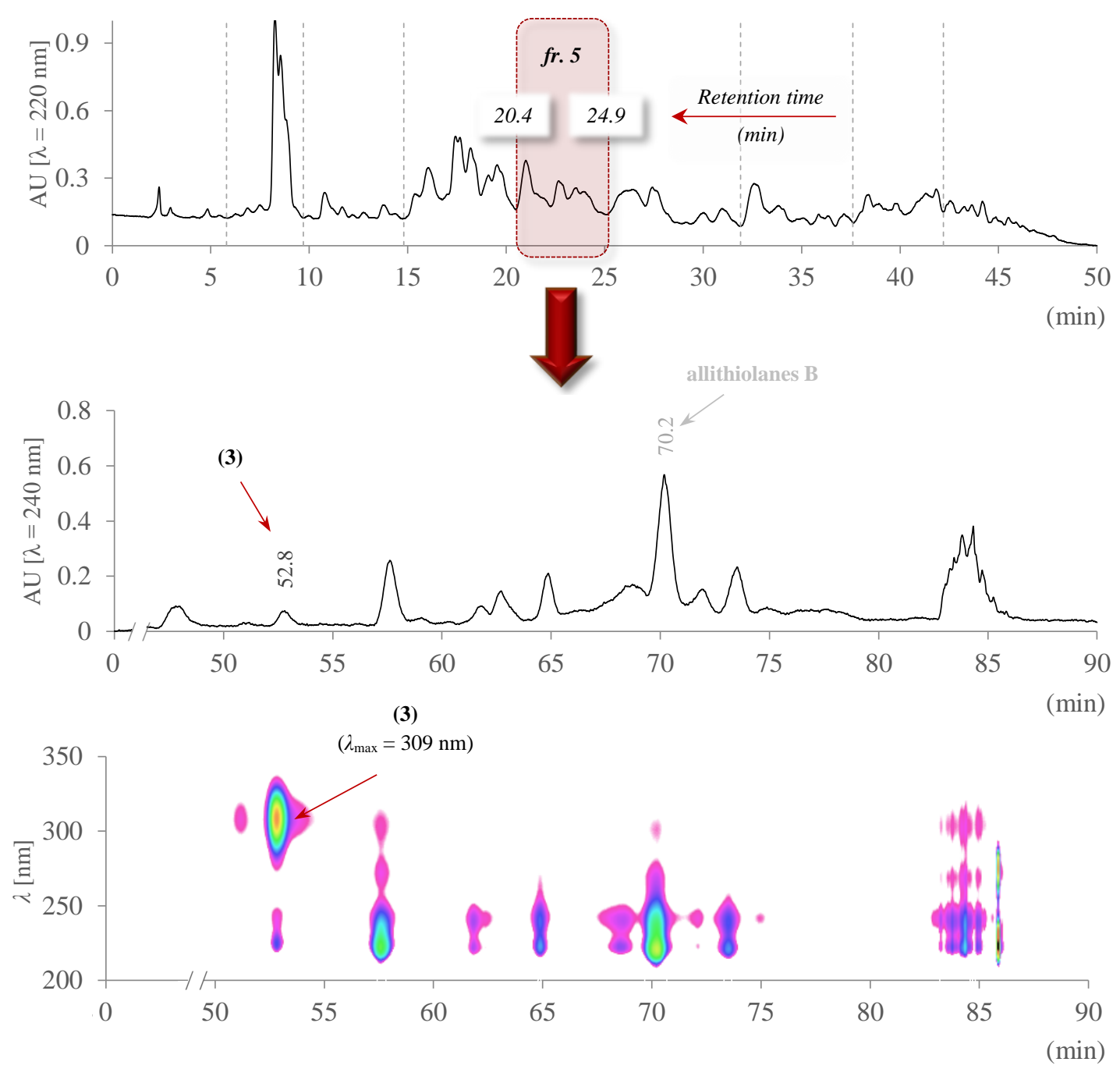

\section{The upper chromatogram:}

Column: $\quad$ Rainin Dynamax-100 $\AA(250 \times 21.4 \mathrm{~mm}, 8 \mu \mathrm{m}$, Varian $)$

Mobile phase: $\quad \mathrm{H}_{2} \mathrm{O}(\mathrm{A})$ and $\mathrm{CH}_{3} \mathrm{CN}(\mathrm{B})$ at $18.0 \mathrm{~mL} \mathrm{~min}^{-1}$

Gradient: $\quad$ A/B 70:30 (0 min), 55:45 (30 min), 5:95 (42 min), 5:95 (48 min), and 70:30 (50 min)

\section{The middle chromatogram:}

Column: $\quad$ Kinetex C-8 $(250 \times 21.2 \mathrm{~mm}, 5 \mu \mathrm{m}$, Phenomenex $)$

Mobile phase: $\quad \mathrm{H}_{2} \mathrm{O}(\mathrm{A})$ and $\mathrm{CH}_{3} \mathrm{CN}(\mathrm{B})$ at $19.1 \mathrm{~mL} \mathrm{~min}^{-1}$

Gradient: $\quad$ A/B 88:12 (0 min), 67:33 (80 $\mathrm{min})$, 5:95 (81 $\mathrm{min})$, 5:95 (89 $\mathrm{min})$, and 88:12 (90 $\mathrm{min})$

\section{The lower chromatogram:}

2D-visualization of the middle chromatogram. 


\section{Isolation of onionins $A_{1-5}$ and $B_{1-3}$ by preparative HPLC}
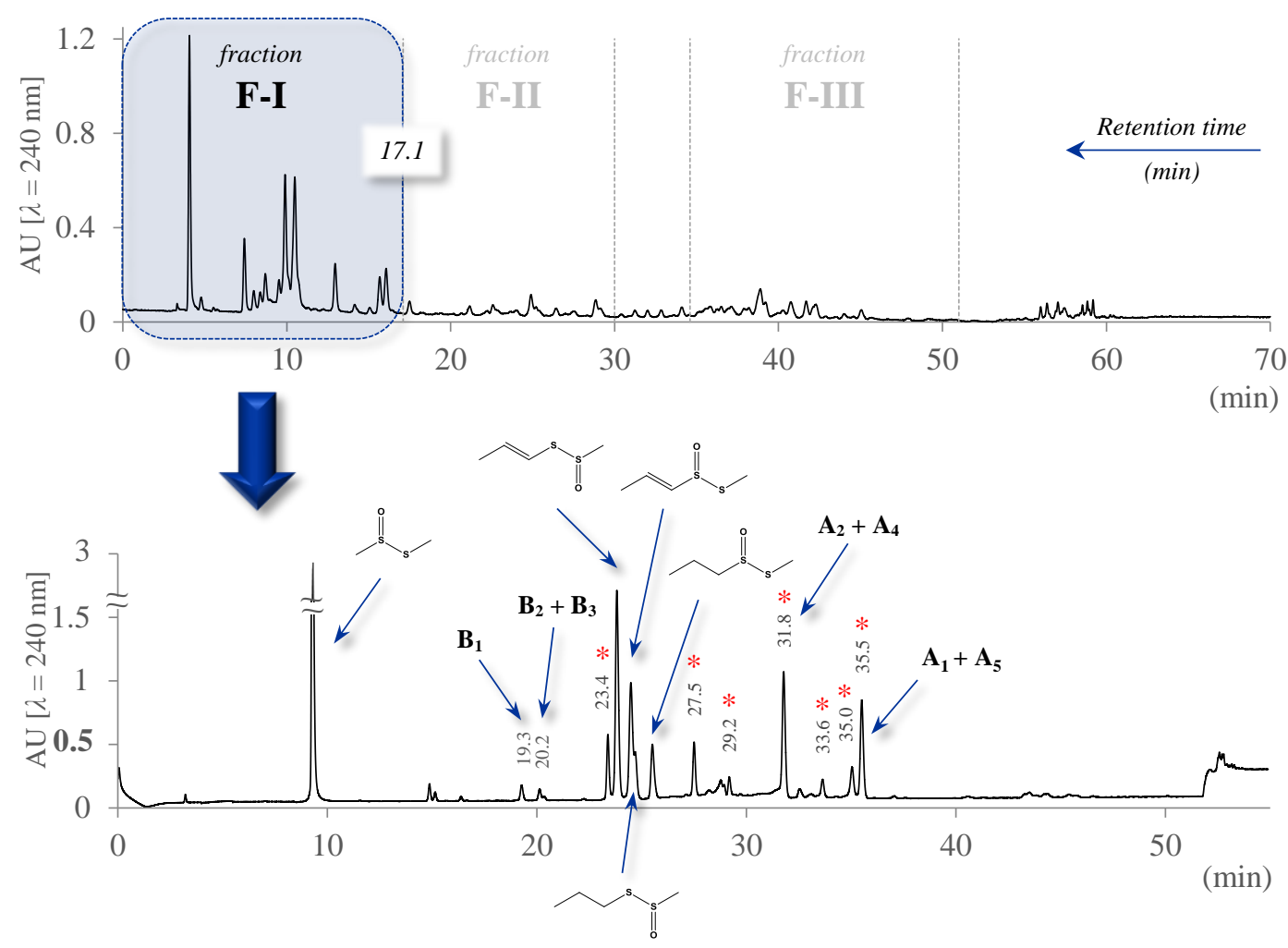

All peaks labeled by $*$ correspond to various stereoisomers of onionin A.

\section{The upper chromatogram:}

Column: $\quad$ Kinetex C-8 $(250 \times 21.2 \mathrm{~mm}, 5 \mu \mathrm{m}$, Phenomenex $)$

Mobile phase: $\quad 0.1 \%$ formic acid in $\mathrm{H}_{2} \mathrm{O}(\mathrm{A})$ and $0.1 \%$ formic acid in $\mathrm{CH}_{3} \mathrm{CN}$ (B) at $19.1 \mathrm{~mL} \mathrm{~min}^{-1}$

Gradient: $\quad$ A/B 85:15 (0 min), 60:40 (50 min), 5:95 (60 min), 5:95 (69 min), and 85:15 (70 min)

\section{The lower chromatogram:}

Column: Kinetex C-8 $(250 \times 21.2 \mathrm{~mm}, 5 \mu \mathrm{m}$, Phenomenex $)$

Mobile phase: $0.1 \%$ formic acid in $\mathrm{H}_{2} \mathrm{O}$ (A) and $0.1 \%$ formic acid in $\mathrm{CH}_{3} \mathrm{CN}$ (B) at $19.1 \mathrm{~mL} \mathrm{~min}^{-1}$

Gradient: A/B 100:0 (0 min), 75:25 (40 min), 5:95 (41 min), 5:95 (49 min), and 100:0 (50 min) 
Isolation of cepathiolanes A1-6 by preparative HPLC

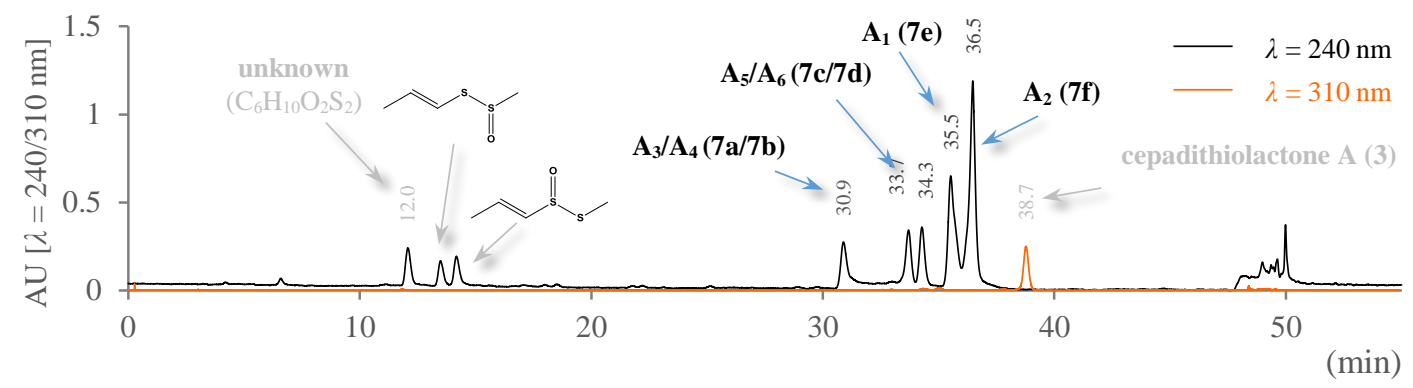

$\begin{array}{ll}\text { Column: } & \text { Kinetex C-8 }(250 \times 21.2 \mathrm{~mm}, 5 \mu \mathrm{m} \text {, Phenomenex }) \\ \text { Mobile phase: } & 0.1 \% \text { formic acid in } \mathrm{H}_{2} \mathrm{O}(\mathrm{A}) \text { and } 0.1 \% \text { formic acid in } \mathrm{CH}_{3} \mathrm{CN}(\mathrm{B}) \text { at } 19.1 \mathrm{~mL} \mathrm{~min}^{-1} \\ \text { Gradient: } & \text { A/B 90:10 (0 min), 65:35 (45 min), 5:95 (46 min), 5:95 (54 min), and 90:10 (55 min) }\end{array}$ 


\section{$\mathrm{UV}$-vis spectrum of cepadithiolactone $\mathrm{A}^{a}$}

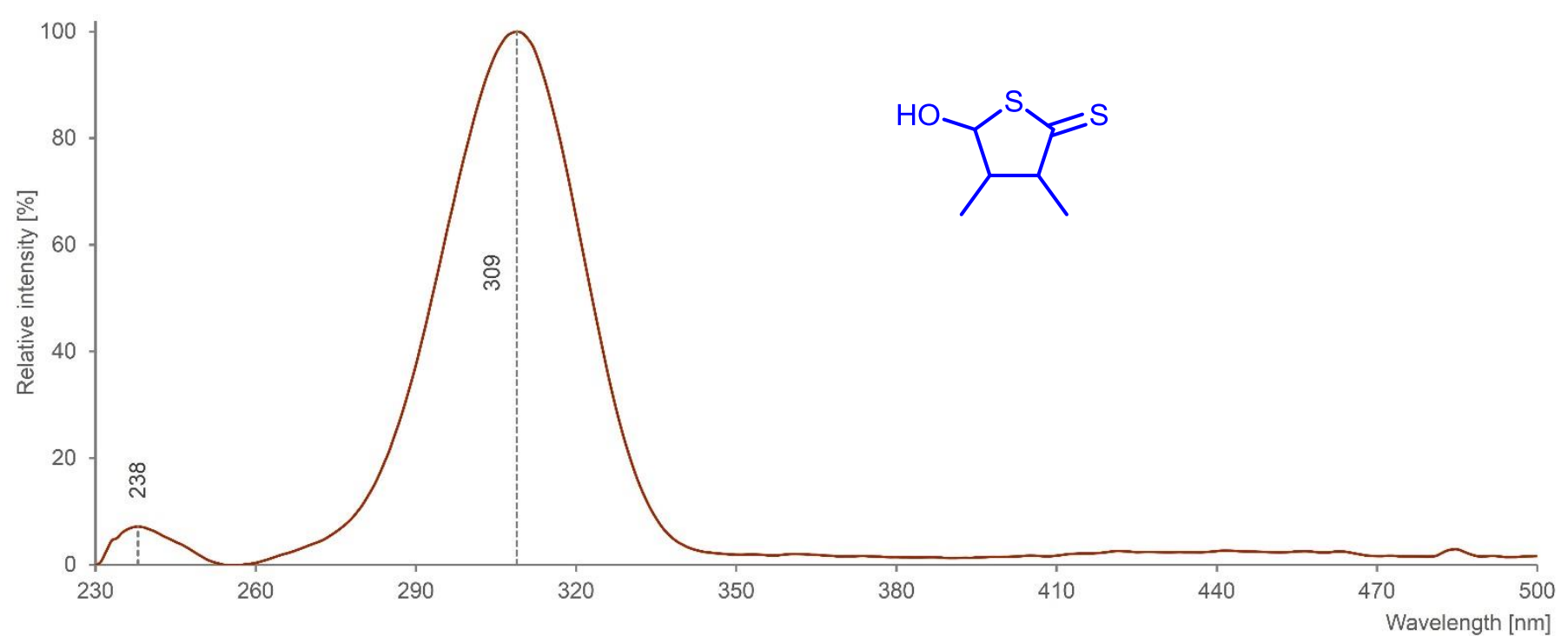

${ }^{a}$ Data retrieved from the PDA detector (in $\mathrm{H}_{2} \mathrm{O} / \mathrm{CH}_{3} \mathrm{CN}, 0.5 \% \mathrm{HCOOH}$ ). 


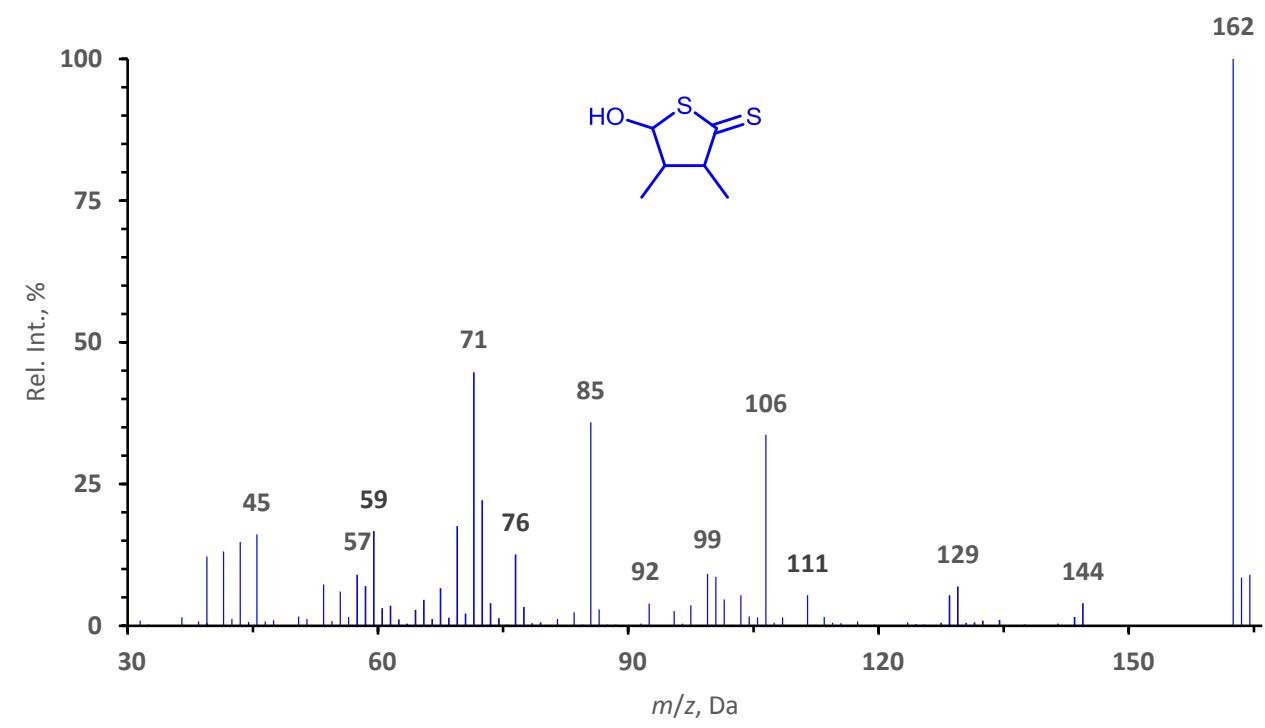

A) El-MS spectrum of cepadithiolactone A (3)

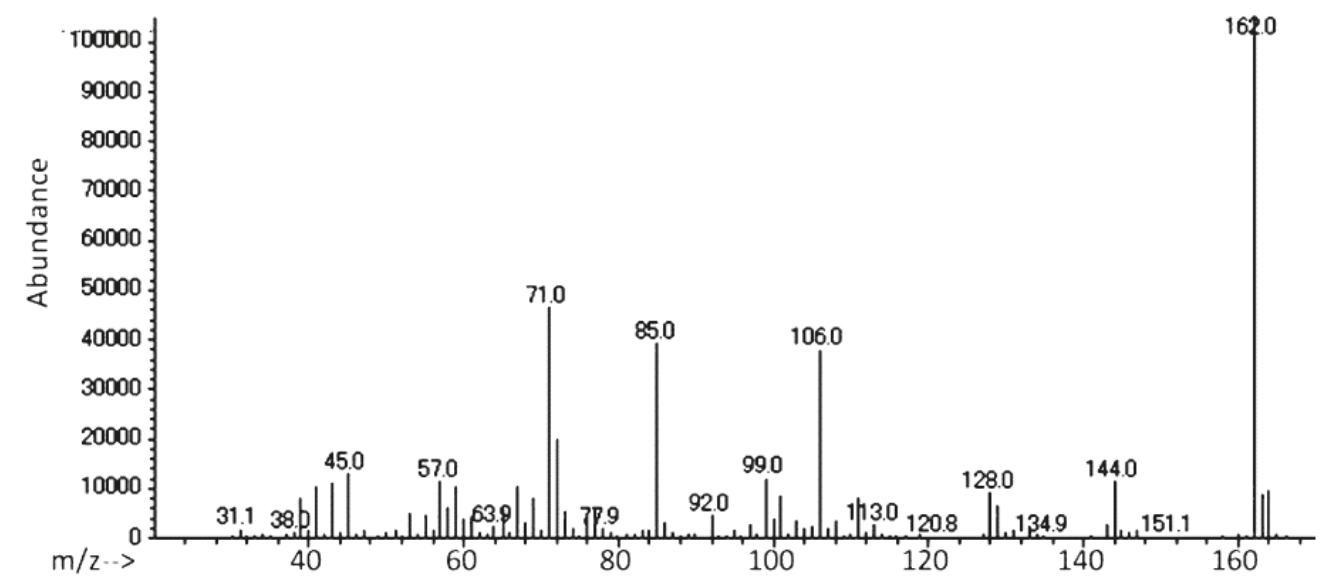

B) EI-MS spectrum of "zwiebelane isomer"

(adopted from Aoyagi et al., J. Agric. Food Chem. 2011, 59, 10893-10900).

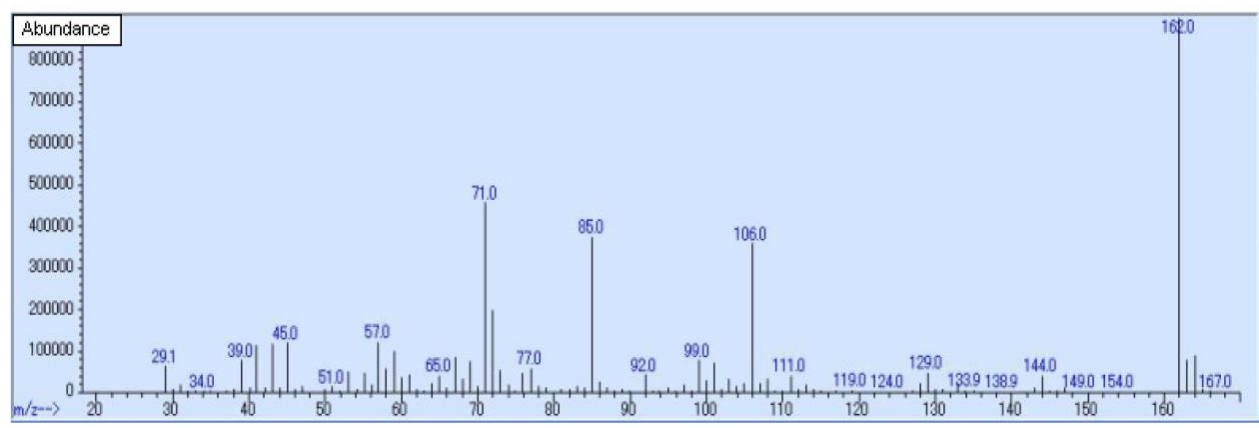

C) EI-MS spectrum of "zwiebelane isomer"

(adopted from Eady et al., Plant Physiol. 2008, 147, 2096-2106). 


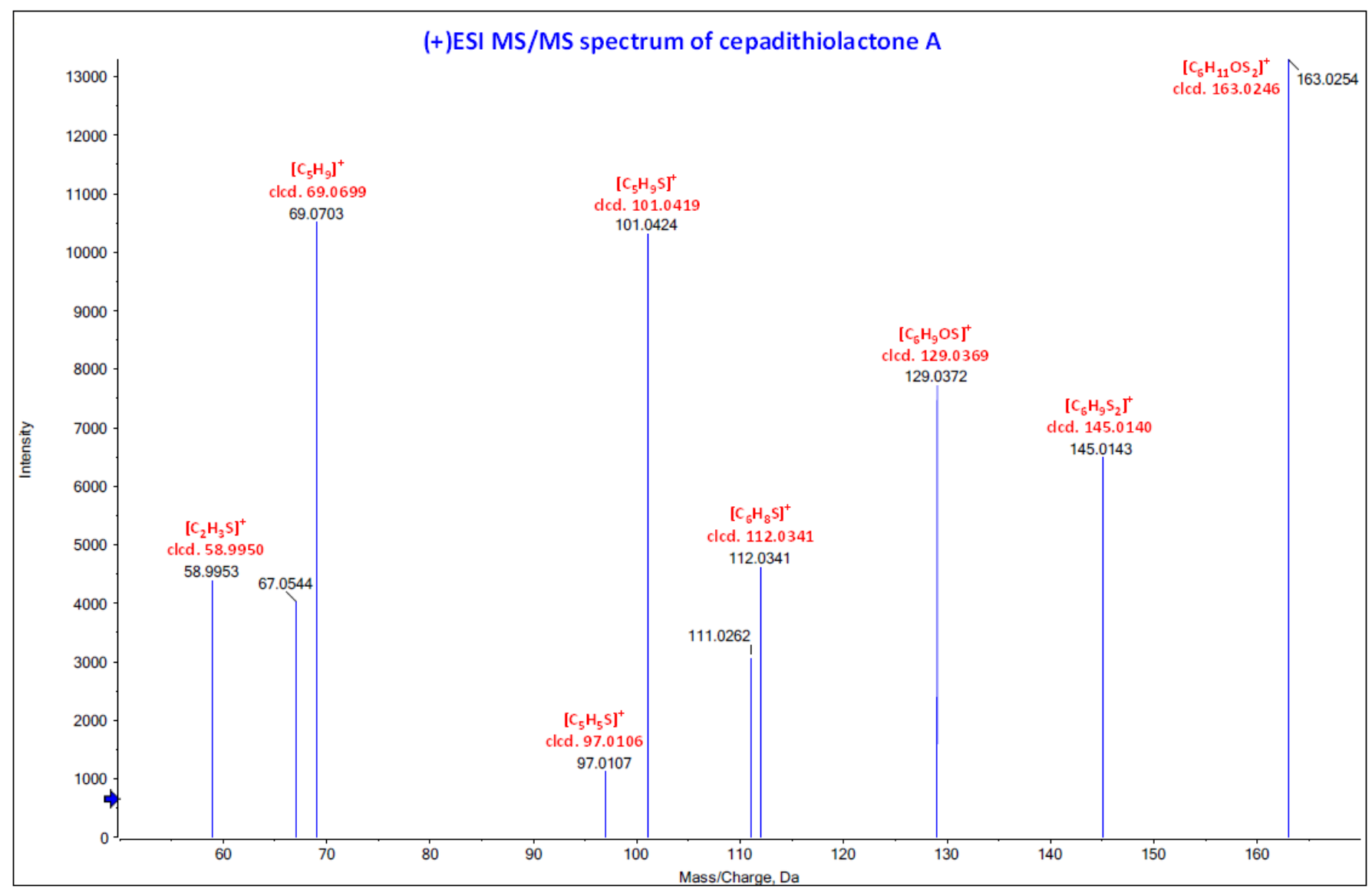

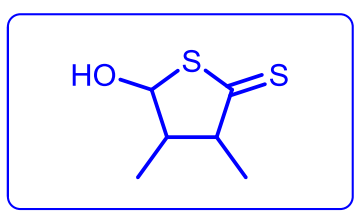

$\mathrm{C}_{6} \mathrm{H}_{10} \mathrm{OS}_{2}$

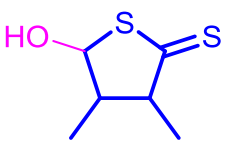

$\left[\mathrm{C}_{6} \mathrm{H}_{9} \mathrm{~S}_{2}\right]^{+}$

$145.0140 \mathrm{Da}$

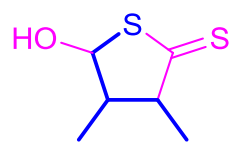

$\left[\mathrm{C}_{5} \mathrm{H}_{9} \mathrm{~S}\right]^{+}$

101.0419 Da

\section{cepadithiolactone $\mathbf{A}$}

$[\mathrm{M}+\mathrm{H}]^{+}$calcd 163.0246 Da

found $163.0248 \mathrm{Da}$

(+)ESI-HRMS - Fragment assignment

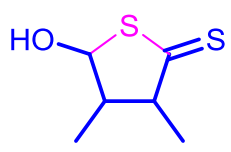

$\left[\mathrm{C}_{6} \mathrm{H}_{9} \mathrm{OS}\right]^{+}$

129.0369 Da

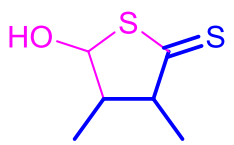

$\left[\mathrm{C}_{5} \mathrm{H}_{5} \mathrm{~S}\right]^{+}$

97.0106 Da<smiles>CC1C(=S)SC(O)C1C</smiles>

$\left[\mathrm{C}_{2} \mathrm{H}_{3} \mathrm{~S}\right]^{+}$

$58.9950 \mathrm{Da}$

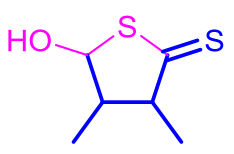

$\left[\mathrm{C}_{6} \mathrm{H}_{8} \mathrm{~S}\right]^{+} \quad\left[\mathrm{C}_{6} \mathrm{H}_{7} \mathrm{~S}\right]^{+}$ 112.0341 Da 111.0263 Da

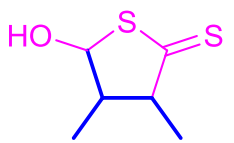

$\left[\mathrm{C}_{5} \mathrm{H}_{9}\right]^{+}$

$69.0699 \mathrm{Da}$ 


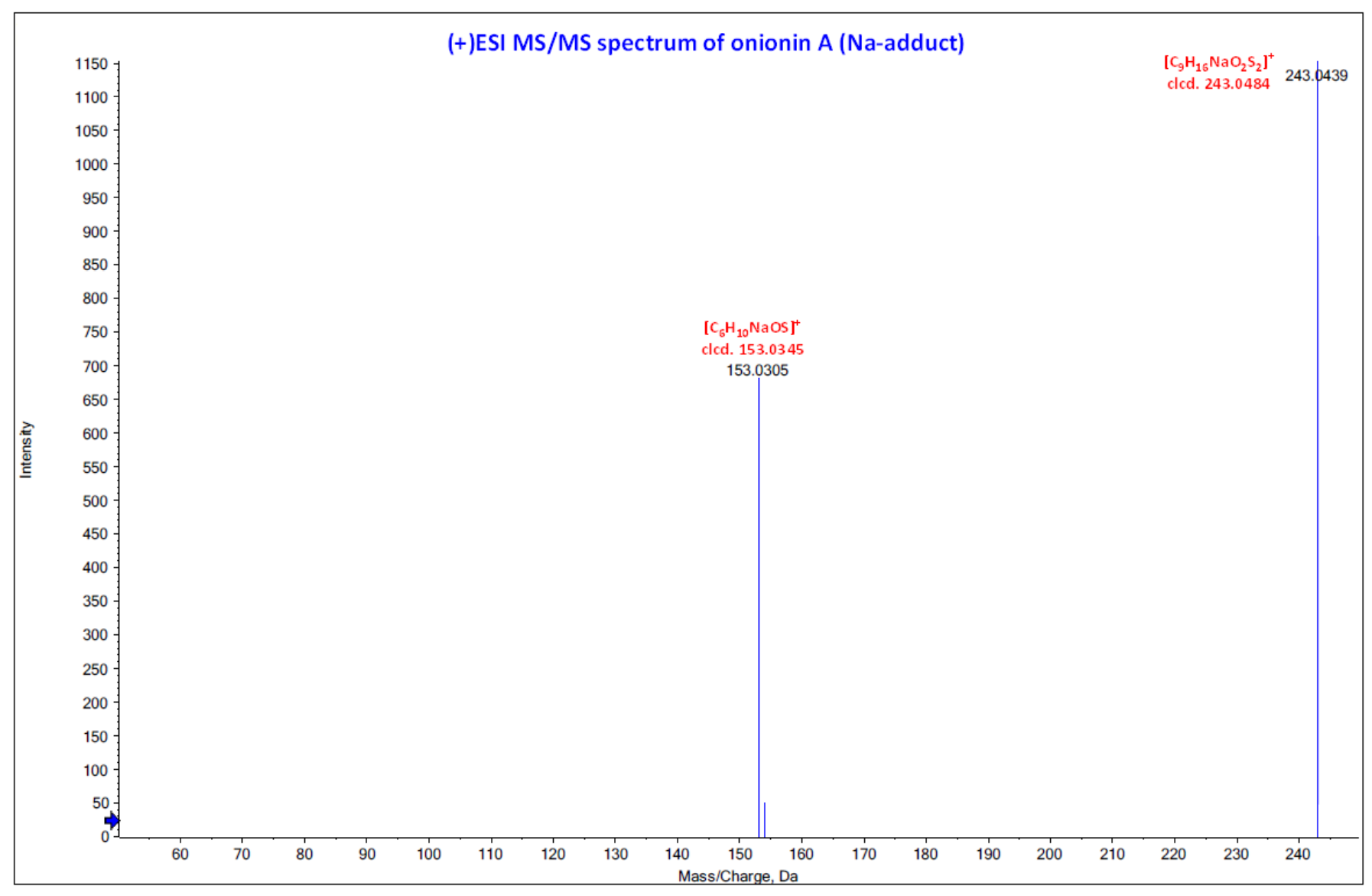

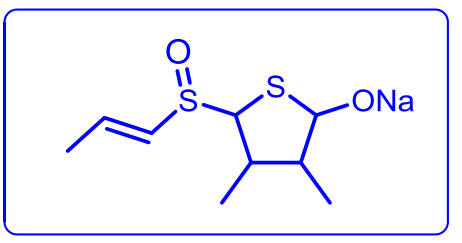

$\mathrm{C}_{9} \mathrm{H}_{15} \mathrm{NaO}_{2} \mathrm{~S}_{2}$

\section{onionin A (Na-adduct)}

$[\mathrm{M}+\mathrm{Na}]^{+}$calcd $243.0484 \mathrm{Da}$

found $243.0480-243.0491 \mathrm{Da}$

(+)ESI-HRMS - Fragment assignment<smiles>CC=CS(=O)C1SC(O[Na])C(C)C1C</smiles>

[C $\left._{6} \mathrm{H}_{10} \mathrm{NaOS}\right]^{+}$

153.0345 Da 


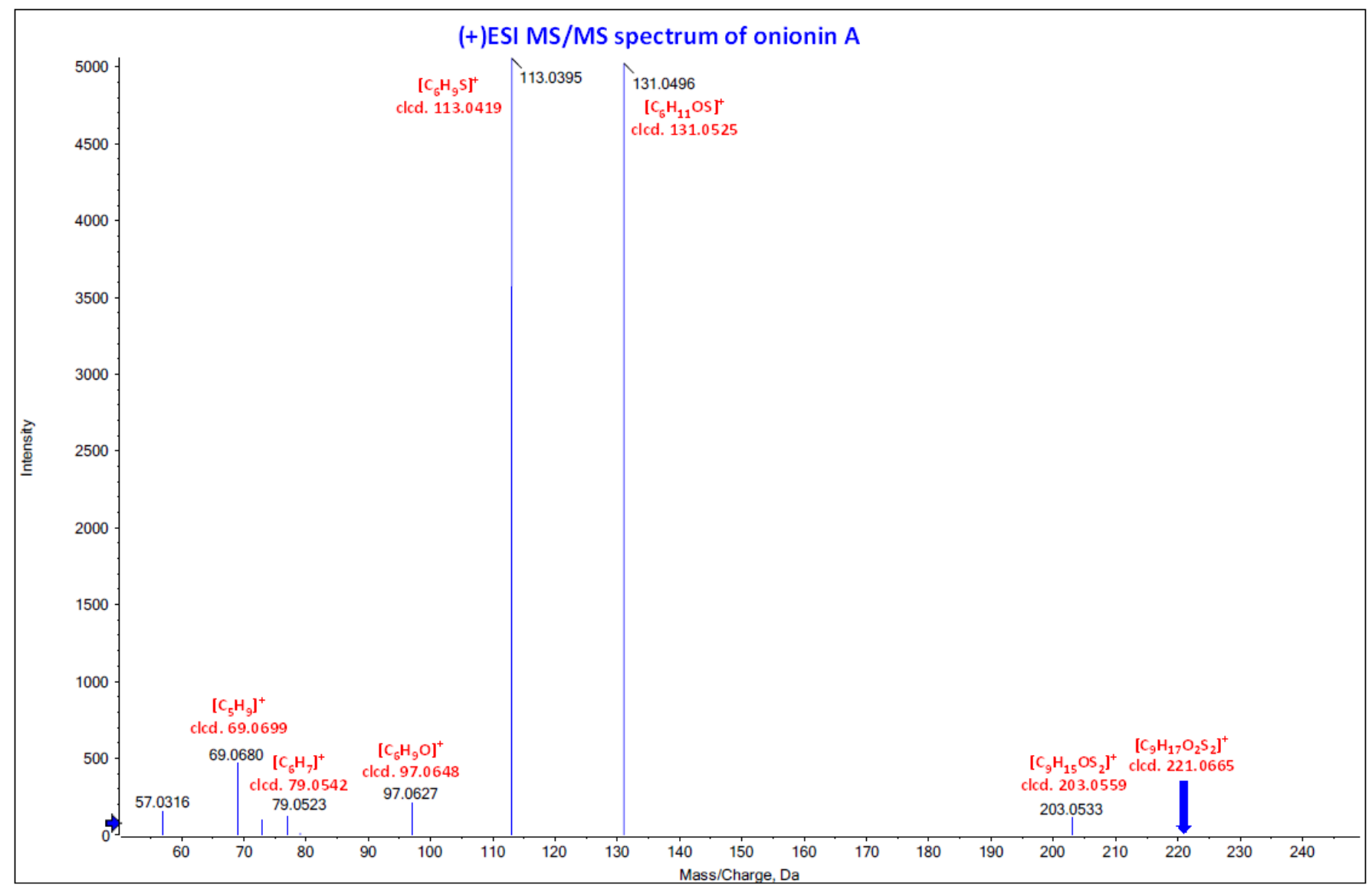

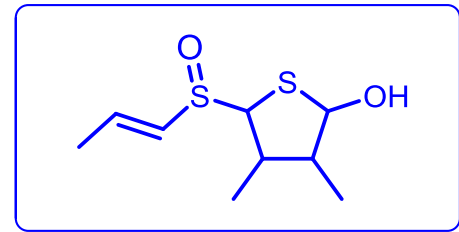

$\mathrm{C}_{9} \mathrm{H}_{16} \mathrm{O}_{2} \mathrm{~S}_{2}$

\section{onionin A}

$[\mathrm{M}+\mathrm{H}]^{+}$calcd $221.0665 \mathrm{Da}$

found 221.0654-221.0660 Da

\section{(+)ESI-HRMS - Fragment assignment}<smiles>CC=CS(=O)C1SC(O)C(C)C1C</smiles>

$\left[\mathrm{C}_{9} \mathrm{H}_{15} \mathrm{OS}_{2}\right]^{+}$ $203.0559 \mathrm{Da}$<smiles>C/C=C/S(=O)C1SC(O)C(C)C1C</smiles>

$\left[\mathrm{C}_{6} \mathrm{H}_{11} \mathrm{OS}\right]^{+}$ $131.0525 \mathrm{Da}$<smiles>C/C=C/S(=O)C1SC(O)C(C)C1C</smiles>

$\left[\mathrm{C}_{6} \mathrm{H}_{9} \mathrm{~S}\right]^{+}$ 113.0419 Da<smiles>CC=CS(=O)C1SC(O)C(C)C1C</smiles>

$\left[\mathrm{C}_{6} \mathrm{H}_{9} \mathrm{O}\right]^{+}$ 97.0648 Da<smiles>C/C=C/S(=O)C1SC(O)C(C)C1C</smiles>

$\left[\mathrm{C}_{6} \mathrm{H}_{9}\right]^{+}$ $79.0542 \mathrm{Da}$<smiles>CC=CS(=O)C1SC(O)C(C)C1C</smiles><smiles>CC=CS(=O)C1SC(O)C(C)C1C</smiles> 


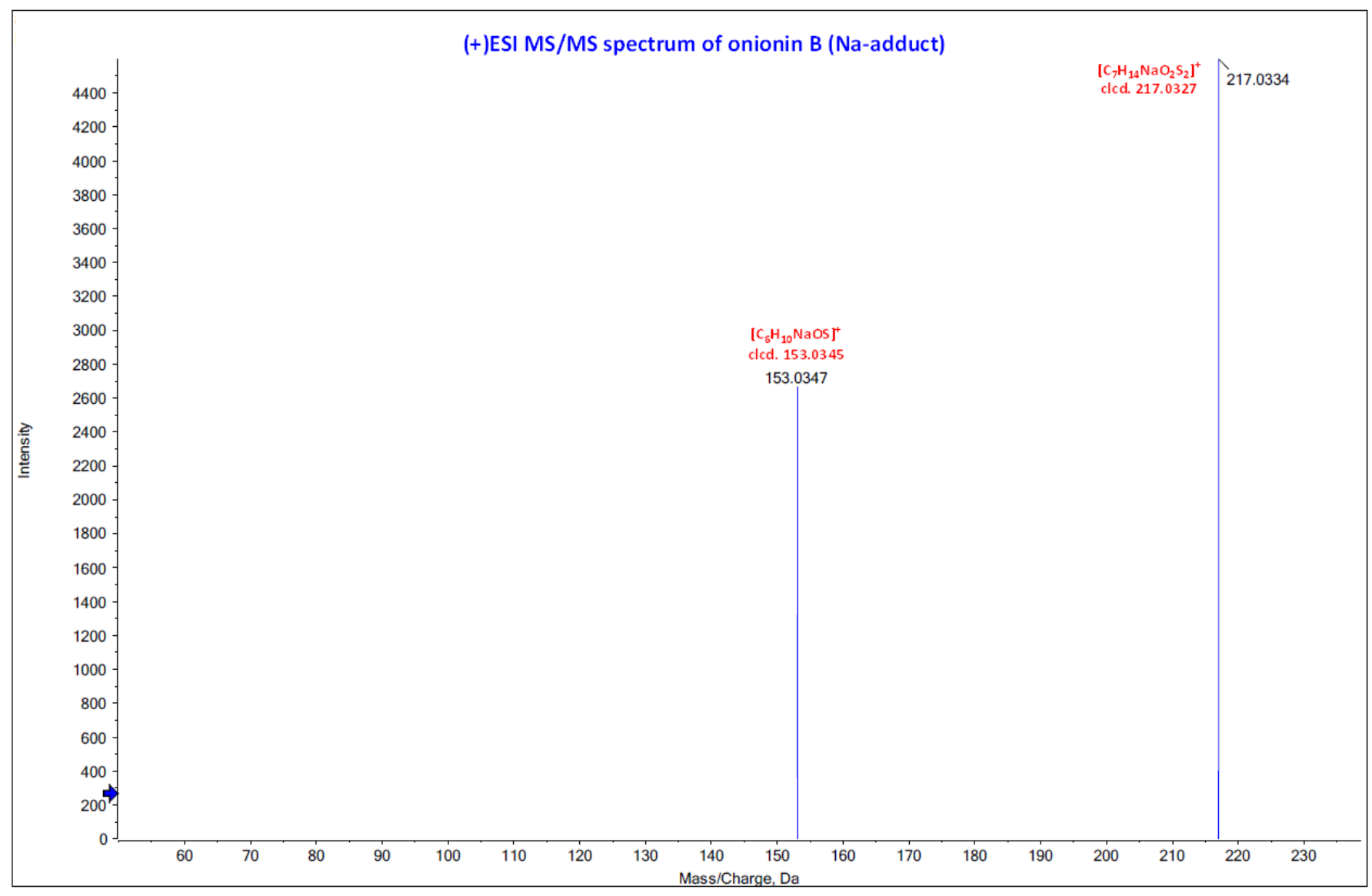

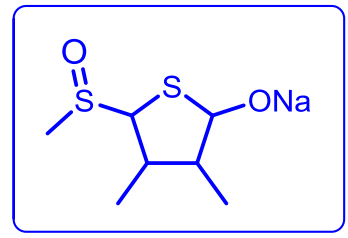

$\mathrm{C}_{7} \mathrm{H}_{13} \mathrm{NaO}_{2} \mathrm{~S}_{2}$

\section{onionin B (Na-adduct)}

$[\mathrm{M}+\mathrm{Na}]^{+}$calcd $217.0327 \mathrm{Da}$

found 217.0318-217.0334 Da

(+)ESI-HRMS - Fragment assignment<smiles>CC1C(O[N+](=O)[O-])SC(S(C)=O)C1C</smiles>

$\left[\mathrm{C}_{6} \mathrm{H}_{10} \mathrm{NaOS}\right]^{+}$

153.0345 Da 


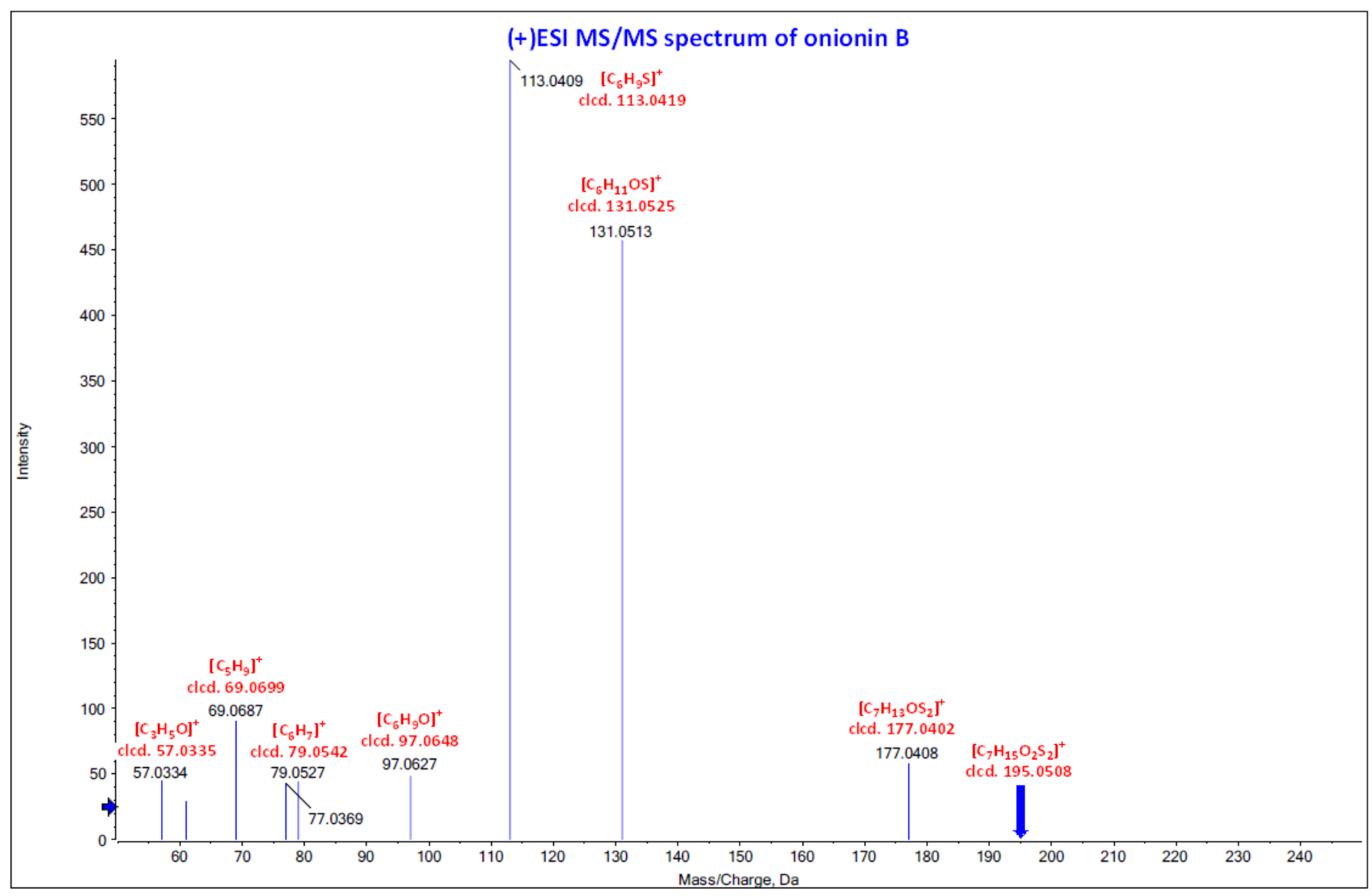

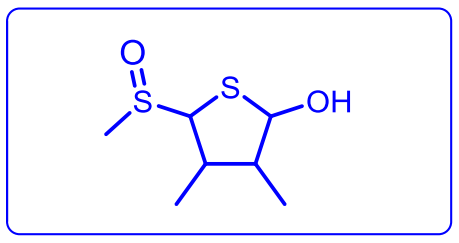

$\mathrm{C}_{7} \mathrm{H}_{14} \mathrm{O}_{2} \mathrm{~S}_{2}$

\section{onionin B}

$[\mathrm{M}+\mathrm{H}]^{+}$calcd $195.0508 \mathrm{Da}$

found 195.0496-195.0502 Da

(+)ESI-HRMS - Fragment assignment<smiles>CC1C(O)SC(S(C)=O)C1C</smiles>

$\left[\mathrm{C}_{7} \mathrm{H}_{13} \mathrm{OS}_{2}\right]^{+}$ $177.0402 \mathrm{Da}$<smiles>CC1C(O)SC(S(C)=O)C1C</smiles>

[C $\left._{6} \mathrm{H}_{11} \mathrm{OS}\right]^{+}$ 131.0525 Da<smiles>CC1C(O)SC(S(C)=O)C1C</smiles>

$\left[\mathrm{C}_{6} \mathrm{H}_{9} \mathrm{~S}\right]^{+}$ 113.0419 Da<smiles>CC1C(O)SC(S(C)=O)C1C</smiles>

$\left[\mathrm{C}_{6} \mathrm{H}_{9} \mathrm{O}\right]^{+}$ $97.0648 \mathrm{Da}$<smiles>CC1C(O)SC(S(C)=O)C1C</smiles><smiles>CC1C(O)SC(S(C)=O)C1C</smiles>

$\left[\mathrm{C}_{6} \mathrm{H}_{9}\right]^{+}$ $79.0542 \mathrm{Da}$<smiles>CC1C(O)SC(S(C)=O)C1C</smiles> 


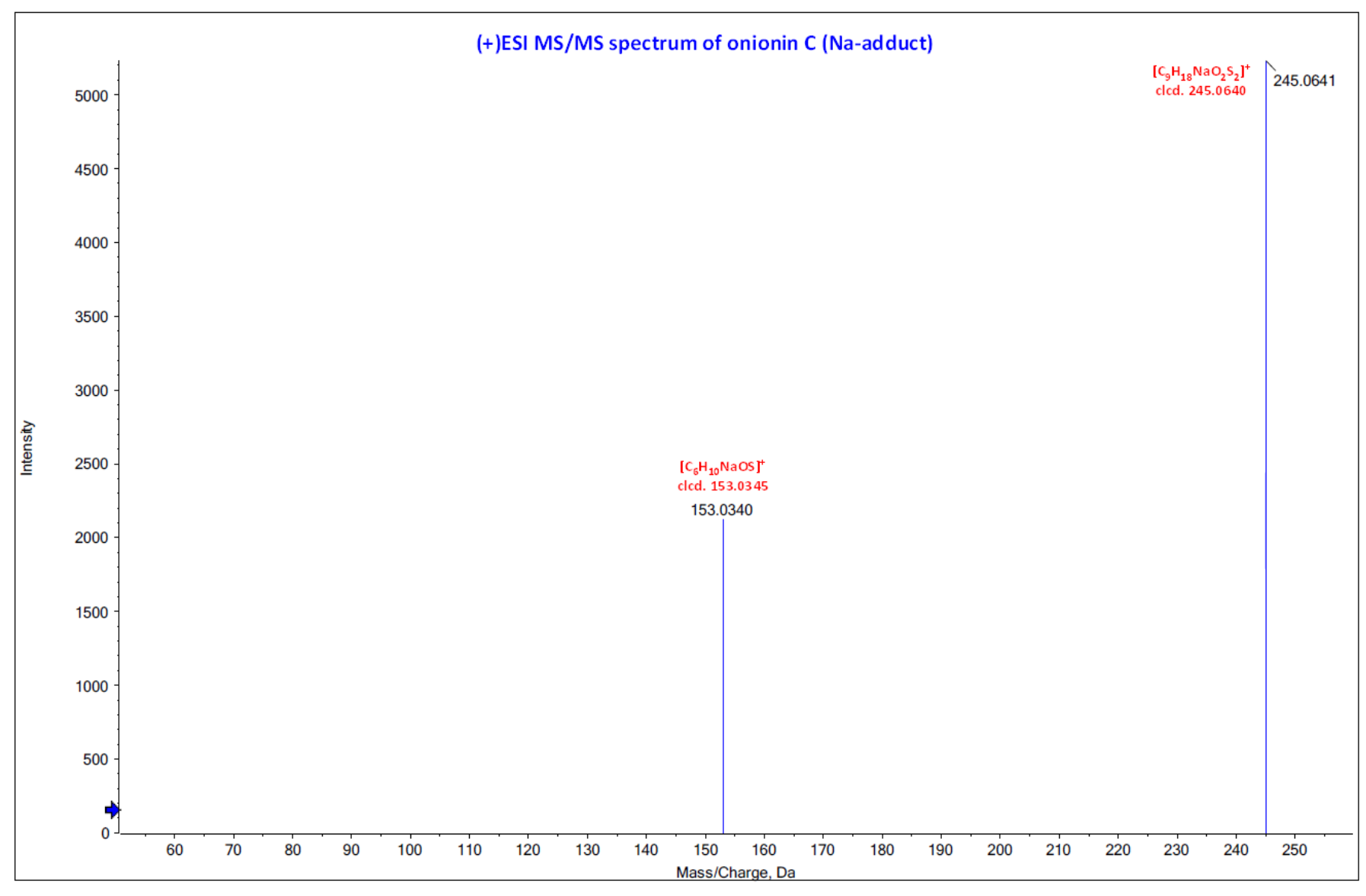

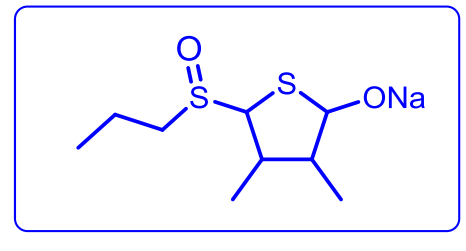

$\mathrm{C}_{9} \mathrm{H}_{17} \mathrm{NaO}_{2} \mathrm{~S}_{2}$

\section{onionin C (Na-adduct)}

$[\mathrm{M}+\mathrm{Na}]^{+}$calcd $245.0640 \mathrm{Da}$

found $245.0630-245.0641 \mathrm{Da}$

(+)ESI-HRMS - Fragment assignment<smiles>CCCS(=O)C1SC(O)C(C)C1C</smiles>

$\left[\mathrm{C}_{6} \mathrm{H}_{10} \mathrm{NaOS}\right]^{+}$

153.0345 Da 


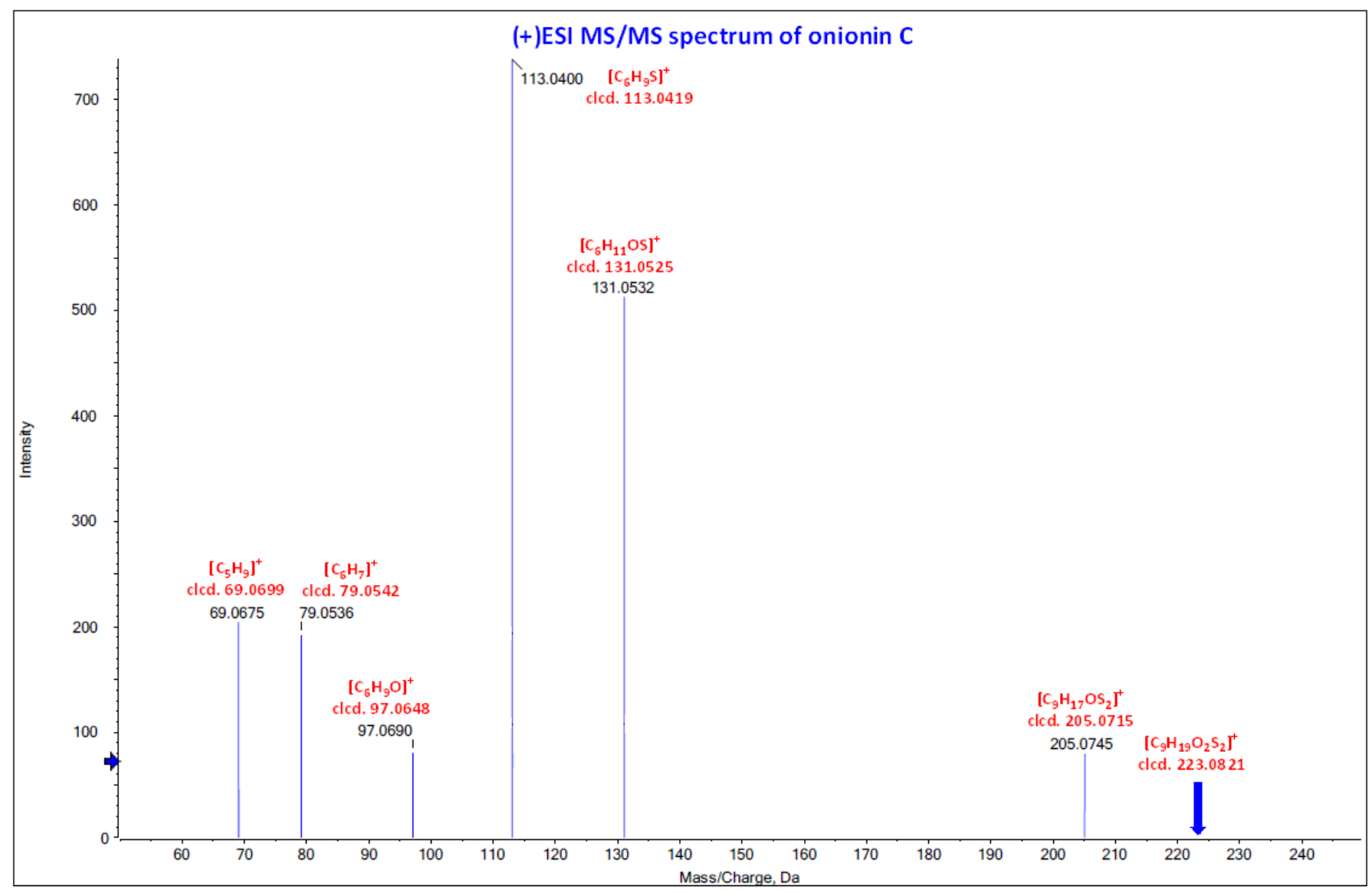

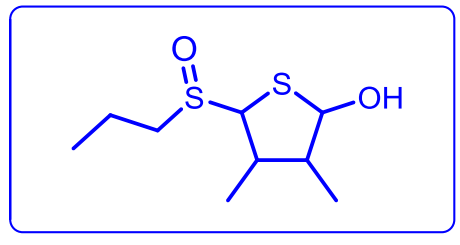

$\mathrm{C}_{9} \mathrm{H}_{18} \mathrm{O}_{2} \mathrm{~S}_{2}$<smiles>CCCS(=O)C1SC(O)C(C)C1C</smiles>

$\left[\mathrm{C}_{9} \mathrm{H}_{17} \mathrm{OS}_{2}\right]^{+}$ $205.0715 \mathrm{Da}$<smiles>CCCS(=O)C1SC(O)C(C)C1C</smiles>

$\left[\mathrm{C}_{6} \mathrm{H}_{9} \mathrm{O}\right]^{+}$ $97.0648 \mathrm{Da}$<smiles>CCCS(=O)C1SC(O)C(C)C1C</smiles>

onionin C

$[\mathrm{M}+\mathrm{H}]^{+}$calcd 223.0821 Da

found 223.0802-223.0817 Da

(+)ESI-HRMS - Fragment assignment<smiles>CCCS(=O)C1SC(O)C(C)C1C</smiles>

$\left[\mathrm{C}_{6} \mathrm{H}_{11} \mathrm{OS}\right]^{+}$ $131.0525 \mathrm{Da}$<smiles>CCCS(=O)C1SC(O)C(C)C1C</smiles>

$\left[\mathrm{C}_{6} \mathrm{H}_{9} \mathrm{~S}\right]^{+}$

113.0419 Da<smiles>CCCS(=O)C1SC(O)C(C)C1C</smiles>

$\left[\mathrm{C}_{6} \mathrm{H}_{9}\right]^{+}$ $79.0542 \mathrm{Da}$<smiles>CCCS(=O)C1SC(O)C(C)C1C</smiles> 


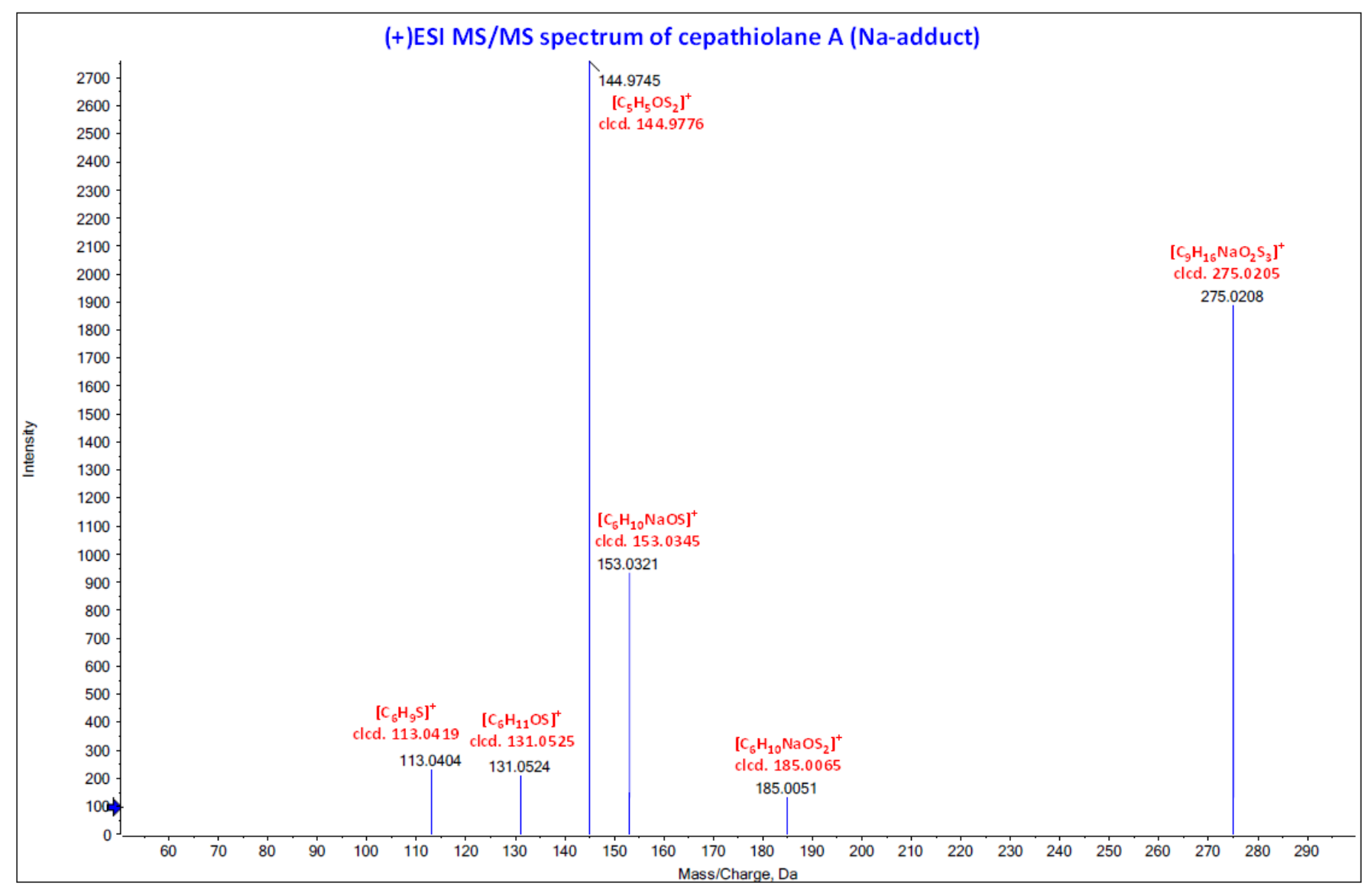

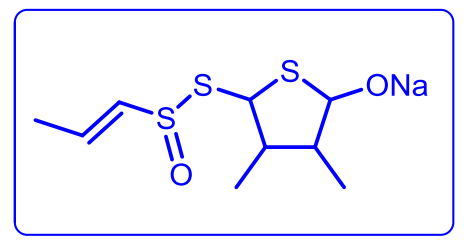

$\mathrm{C}_{9} \mathrm{H}_{15} \mathrm{NaO}_{2} \mathrm{~S}_{3}$

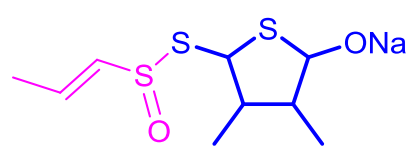

$\left[\mathrm{C}_{6} \mathrm{H}_{10} \mathrm{NaOS}_{2}\right]^{+}$

185.0065 Da

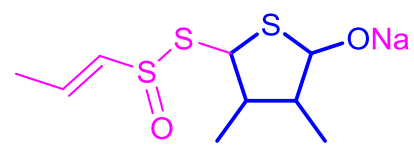

[C $\left._{6} \mathrm{H}_{11} \mathrm{OS}\right]^{+}$

$131.0525 \mathrm{Da}$

\section{cepathiolane A (Na-adduct)}

$[\mathrm{M}+\mathrm{Na}]^{+}$calcd $275.0205 \mathrm{Da}$

found $275.0196-275.0208 \mathrm{Da}$

\section{(+)ESI-HRMS - Fragment assignment}

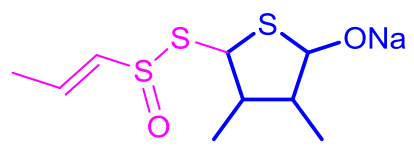

[C $\left._{6} \mathrm{H}_{10} \mathrm{NaOS}\right]^{+}$ 153.0345 Da<smiles>CC=CS(=O)SC1SC([AlH2])C(C)C1C</smiles>

$\left[\mathrm{C}_{6} \mathrm{H}_{9} \mathrm{~S}\right]^{+}$

113.0419 Da<smiles>CC=CS(=O)SC1SC(O[Na])C(C)C1C</smiles>

$\left[\mathrm{C}_{5} \mathrm{H}_{5} \mathrm{OS}_{2}\right]^{+}$ 144.9776 Da 


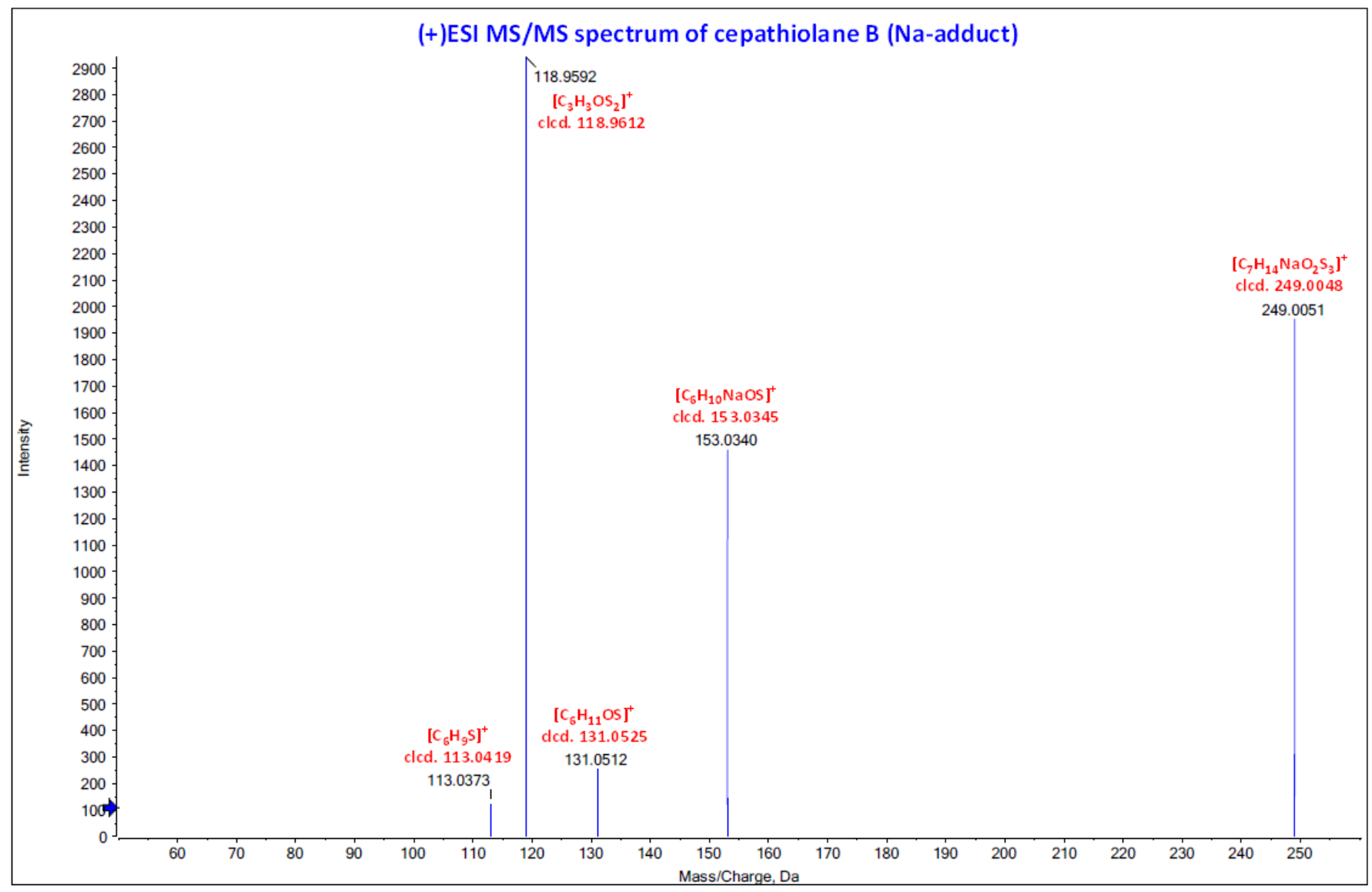

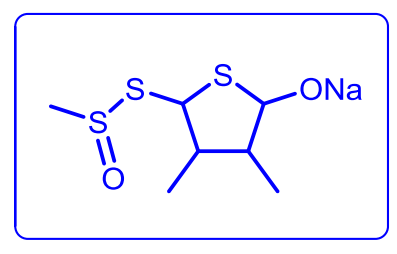

$\mathrm{C}_{7} \mathrm{H}_{13} \mathrm{NaO}_{2} \mathrm{~S}_{3}$

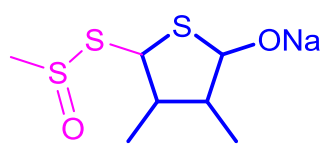

[C $\left._{6} \mathrm{H}_{10} \mathrm{NaOS}\right]^{+}$

153.0345 Da

\section{cepathiolane B (Na-adduct)}

$[\mathrm{M}+\mathrm{Na}]^{+}$calcd $249.0048 \mathrm{Da}$

found $249.0040-249.0059 \mathrm{Da}$

\section{(+)ESI-HRMS - Fragment assignment}

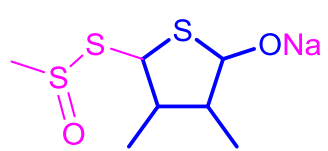

[C $\left._{6} \mathrm{H}_{11} \mathrm{OS}\right]^{+}$

131.0525 Da

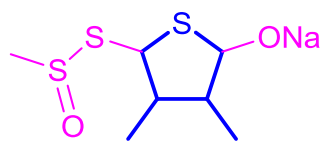

$\left[\mathrm{C}_{6} \mathrm{H}_{9} \mathrm{~S}\right]^{+}$

113.0419 Da

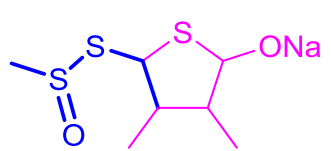

$\left[\mathrm{C}_{3} \mathrm{H}_{3} \mathrm{OS}_{2}\right]^{+}$ 118.9612 Da 


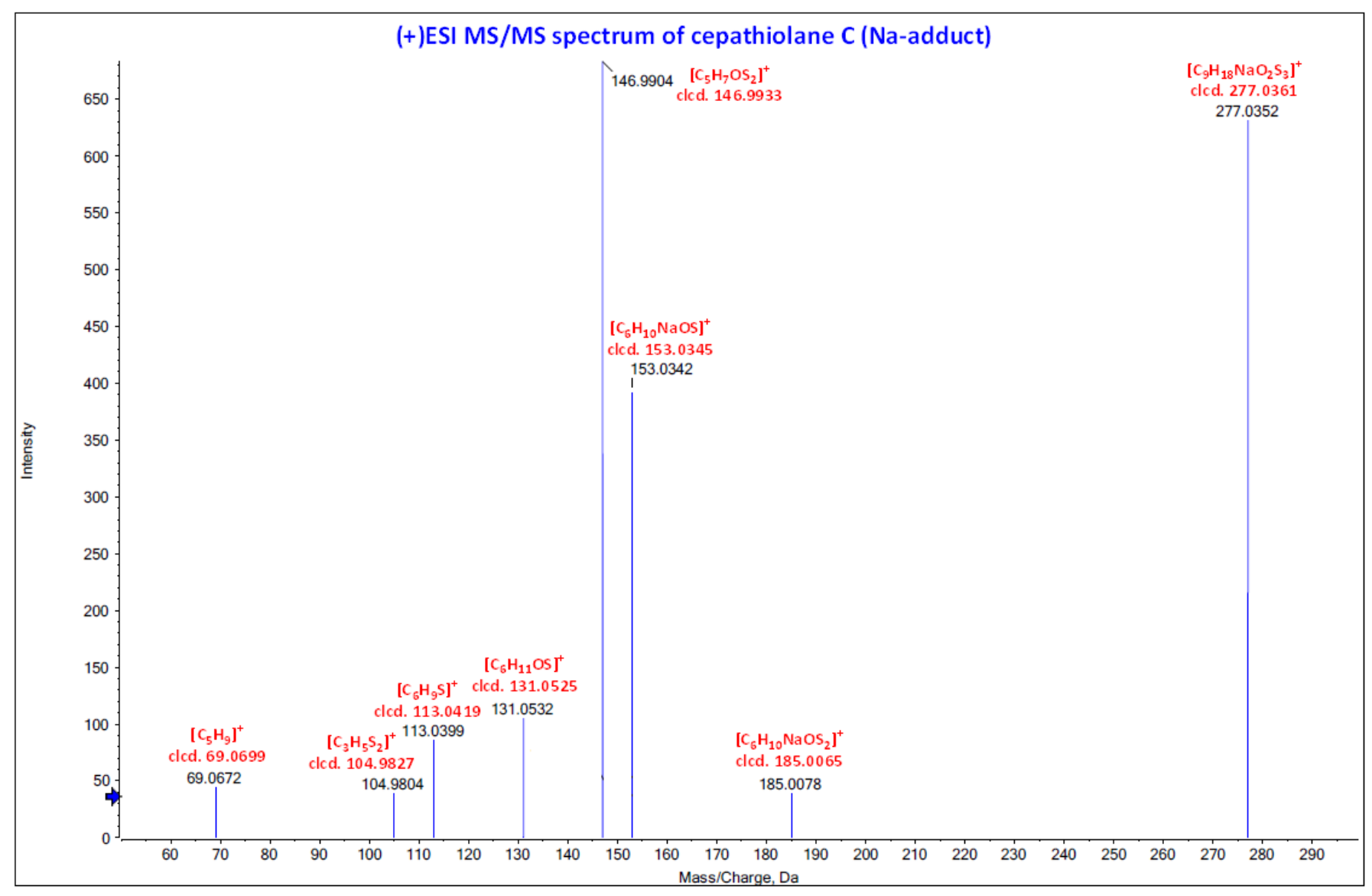

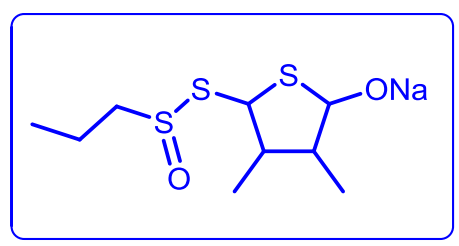

$\mathrm{C}_{9} \mathrm{H}_{17} \mathrm{NaO}_{2} \mathrm{~S}_{3}$

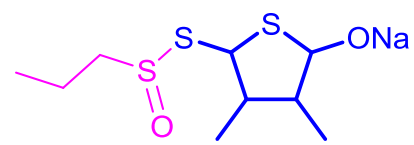

$\left[\mathrm{C}_{6} \mathrm{H}_{10} \mathrm{NaOS}_{2}\right]^{+}$

$185.0065 \mathrm{Da}$

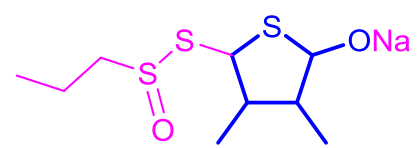

$\left[\mathrm{C}_{6} \mathrm{H}_{11} \mathrm{OS}\right]^{+}$

$131.0525 \mathrm{Da}$

\section{cepathiolane $\mathrm{C}$ (Na-adduct)}

$[\mathrm{M}+\mathrm{Na}]^{+}$calcd $277.0361 \mathrm{Da}$

found $277.0352-277.0371 \mathrm{Da}$

(+)ESI-HRMS - Fragment assignment

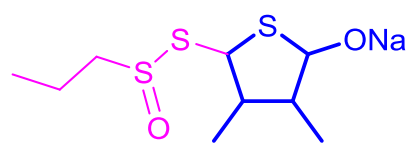

$\left[\mathrm{C}_{6} \mathrm{H}_{10} \mathrm{NaOS}\right]^{+}$

153.0345 Da

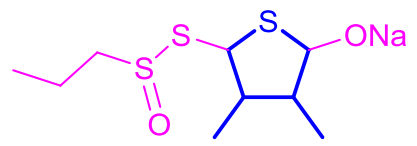

$\left[\mathrm{C}_{6} \mathrm{H}_{9} \mathrm{~S}\right]^{+}$

113.0419 Da<smiles>CCCS(=O)SC1SC(O[N+](=O)[O-])C(C)C1C</smiles>

$\left[\mathrm{C}_{5} \mathrm{H}_{7} \mathrm{OS}_{2}\right]^{+}$ 146.9933 Da 
IR spectra of cepadithiolactone $A$, onionins $A_{2}+A_{4}$, and cepathiolanes $A_{1}+A_{2}$.
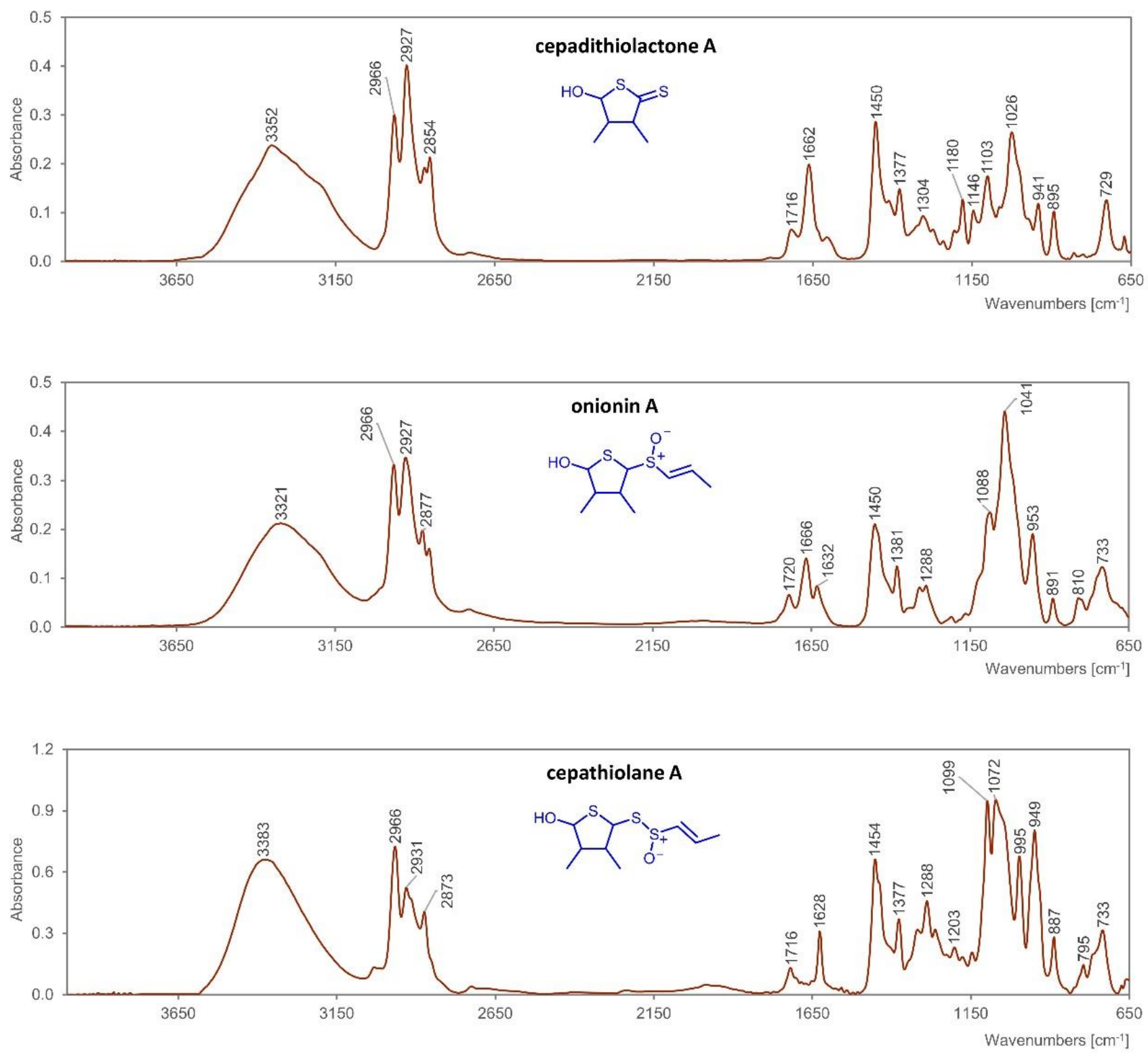


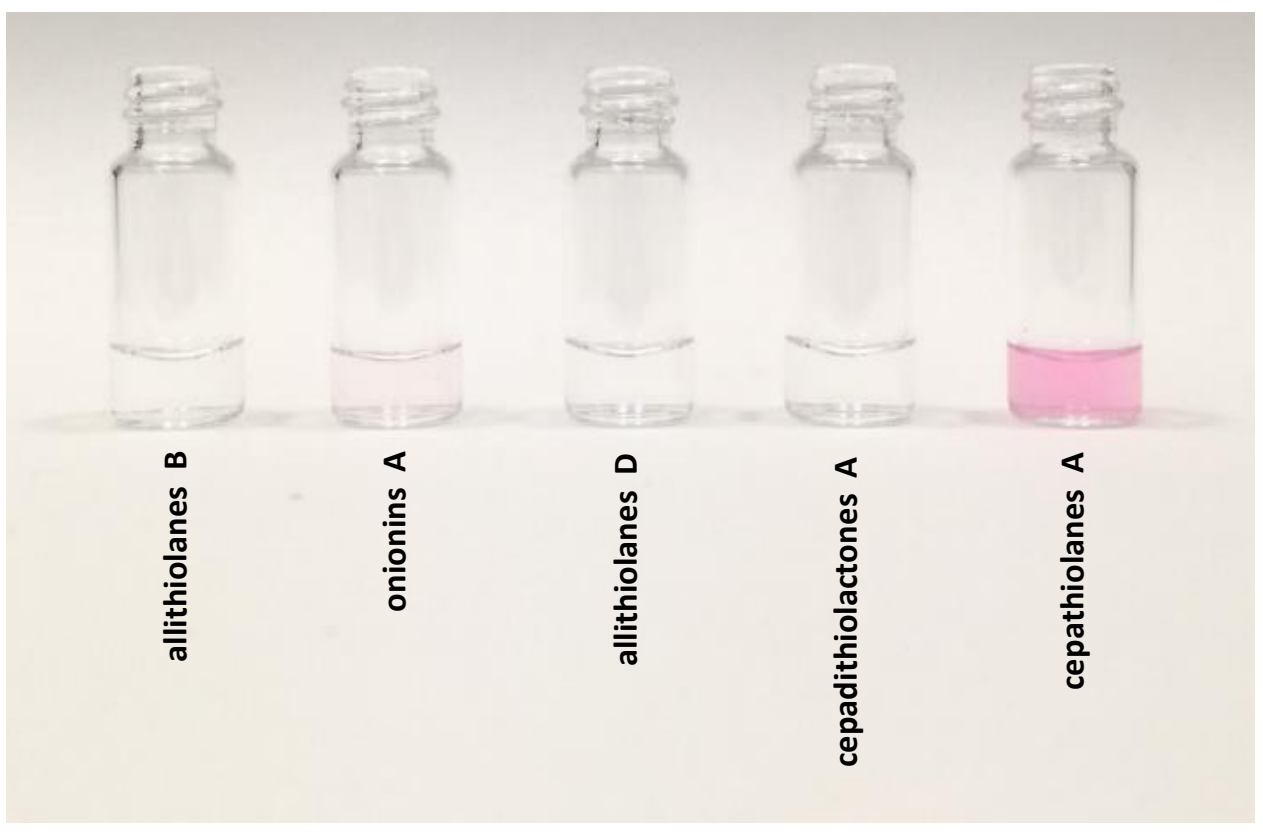

The result of the pinking assay. 